\title{
Antithrombotics in trauma: management strategies in the older patients
}

\author{
Henna Wong ${ }^{1,2}$ \\ Nicola Lovett ${ }^{3}$ \\ Nicola Curry' \\ Ku Shah ${ }^{2}$ \\ Simon J Stanworth ${ }^{1,2,4}$ \\ 'Department of Clinical Haematology, \\ Oxford University Hospitals \\ NHS Foundation Trust, ${ }^{2}$ Radcliffe \\ Department of Medicine, University \\ of Oxford, Oxford BRC Haematology \\ Theme, ${ }^{3}$ Department of Geratology, \\ Oxford University Hospitals NHS \\ Foundation Trust, ${ }^{4}$ Department \\ of Haematology, NHS Blood and \\ Transplant, John Radcliffe Hospital, \\ Oxford University Hospitals NHS \\ Foundation Trust, Oxford, UK
}

This article was published in the following Dove Press journal: Journal of Blood Medicine

4 October 2017

Number of times this article has been viewed

\begin{abstract}
The ageing population has resulted in a change in the demographics of trauma, and older adult trauma now accounts for a growing number of trauma admissions. The management of older adult trauma can be particularly challenging, and exhibits differences to that of the younger age groups affected by trauma. Frailty syndromes are closely related with falls, which are the leading cause of major trauma in older adults. Comorbid disease and antithrombotic use are more common in the older population. Physiological changes that occur with ageing can alter the expected clinical presentation of older persons after injury and their susceptibility to injury. Following major trauma, definitive control of hemorrhage remains essential for improving outcomes. In the initial assessment of an injured patient, it is important to consider whether the patient is taking anticoagulants or antiplatelets and if measures to promote hemostasis such as reversal are indicated. After hemostasis is achieved and bleeding has stopped, longer-term decisions to recommence antithrombotic agents can be challenging, especially in older people. In this review, we discuss one aspect of management for the older trauma patients in greater detail, that is, acute and longer-term management of antithrombotic therapy. As we consider the health needs of an ageing population, rise in elderly trauma and increasing use of antithrombotic therapy, the need for research in this area becomes more pressing to establish best practice and evidence-based care.
\end{abstract}

Keywords: injury, elderly, anticoagulation, antiplatelet, antithrombotic

\section{Case history}

An 83-year-old lady presents with headache and acute confusion following a fall. Her Glasgow Coma Scale (GCS) is 14/15. She takes warfarin for atrial fibrillation (AF) and has no other past medical history.

What are the differential diagnoses? When might reversal of warfarin be considered?

If she has an intracranial hemorrhage, what would be the acute management?

In the longer term, which factors need consideration when deciding on future anticoagulation?

\section{Introduction}

Correspondence: Henna Wong

Oxford Haemophilia and Thrombosis

Centre, Churchill Hospital, Old Road,

Oxford OX3 7LE, UK

Tel +44 I865225300

Fax +44 I865 225608

Email henna.wong@ouh.nhs.uk

The ageing population has resulted in a change in the demographics of trauma. This includes an increase in clinically vulnerable older people with comorbid disease, functional dependence, disability and frailty syndromes. ${ }^{1}$ Many of these patients are undergoing anticoagulation or antiplatelet therapy and are also at high risk of falls and sustaining injury with potentially serious hemorrhagic complications. Diminished 
physiological reserve and increased comorbidities may mean older people are more susceptible to injury and bleeding. Timely reversal of anticoagulation in the acute setting can help restore hemostatic function and potentially reduce bleeding, prior to definitive surgery or other interventions. However, once hemostasis is achieved, decisions to recommence antithrombotic agents in the longer term, weighing up bleeding and thrombotic risk, can be challenging. The aims of this article are to review the evidence and strategies of managing oral anticoagulation and antiplatelet therapy in the older patients in the acute setting and longer term after major trauma.

\section{Trauma in the older population: the changing landscape of trauma}

For the purposes of this review, we refer to older as those aged over 65, as many of the published studies on elderly trauma use this age threshold. Major trauma is defined as an injury or combination of injuries that are life-threatening and may result in long-term disability. ${ }^{2}$ Trauma in the older population now accounts for a growing number of major trauma admissions to hospital. ${ }^{3}$ As life expectancy increases and the population ages, these numbers are set to rise. ${ }^{4-7}$ In countries such as the UK, it is not uncommon to see patients in their 80s and 90s taking anticoagulants or antiplatelets admitted with trauma.

Despite similar injury severity, older people have poorer outcomes than younger people. ${ }^{8}$ The reasons for this are unclear. Physiological changes in the cardiovascular and respiratory systems that occur with ageing mean older people are less likely to be able to tolerate and compensate for acute blood loss. Vital signs are less reliable, as "normal blood pressure and heart rate" are not necessarily reassuring. An observational study showed occult hypoperfusion was present in around $20 \%$ of older trauma patients. ${ }^{9}$ This may lead to potential under-triage and delay in recognition of injury severity. In addition, the GCS may not be as reliable a marker of brain injury in older people as in the young. ${ }^{10,11}$ Furthermore, the incidence of cardiovascular and thrombotic disease rises in the older population, and older people are more likely to take anticoagulants or antiplatelets for primary or secondary prophylaxis. ${ }^{12-14}$

\section{Antithrombotic use in the older population}

The prevalence of AF increases with age: 3.8\% among those aged over 60 years rising to $9.0 \%$ among those aged over 80 years. ${ }^{15}$ Treatment with an oral anticoagulant, traditionally warfarin, has been shown to be highly effective, reducing the relative risk of stroke in patients with AF by around twothirds. ${ }^{16,17}$ More recently, trials comparing warfarin and direct oral anticoagulants (DOACs) for non-valvular AF have shown similar stroke prevention efficacy but a lower incidence of intracranial bleeding with the DOACs. ${ }^{18,19}$ Current guidelines recommend anticoagulation for stroke prevention for patients over the age of 75 with AF or over 65 with additional risk factors. ${ }^{20}$

Studies show $40-66 \%$ of adults aged 75 years or older in the USA and Europe take 1 or more antiplatelet agents with about half of these indicated for lifelong secondary prevention. ${ }^{21,22}$ The use and choice of DOACs has also increased, ${ }^{23}$ and it is expected that this increased usage will also be reflected in the older trauma population. Although these agents have proven overall benefit for patients at risk of thromboembolic events, they have not been well studied in frail patients; not uncommonly, the studies of DOACs and warfarin did not include the oldest-old and/or excluded those at risk of falling. ${ }^{18,19,24}$ Therefore, some physicians may be reluctant to prescribe anticoagulants, especially for the very old or frailest patients, as they are perceived to be at higher risk for the consequences of falls such as traumatic brain injury (TBI). Although these consequences are rare, they can have devastating effects and are therefore likely to influence clinical decision making.

\section{Frailty and falls}

Frailty is an important factor in trauma. It is defined as a syndrome of decreased physiological reserve and resistance to stressors, which results in increased vulnerability to poor health outcomes and mortality. ${ }^{25}$ Two main models of frailty have emerged: the phenotype model ${ }^{26}$ and the clinical deficit model. ${ }^{27}$ The phenotype model describes a group of patient characteristics centered on sarcopenia (unintentional weight loss, reduced muscle strength, reduced gait speed, self-reported exhaustion and low energy expenditure). ${ }^{28}$ The clinical deficit model is based on a score of long-term conditions or impairments, where the higher the score the greater the risk of an adverse outcome. An assessment of frailty is a key part of a comprehensive assessment of an older person. After injury, a frailty assessment can aid clinical decisionmaking for thromboprophylaxis by helping to understand vulnerability and risk of poor outcomes.

The risk of falls is increased in older people with frailty, and they have worse outcomes after trauma. ${ }^{25,29}$ Falls are the primary cause of trauma in older age, with at least one-third of people over the age of 65 having at least 1 fall a year. ${ }^{30}$ 
The highest rates of falls are seen among the frail and those in nursing homes. The reasons for falls are often multifactorial, and risk factors include polypharmacy, sarcopenia, poor balance, visual impairment, arthritis, depression and dementia. Falls in older people are more likely to result in injuries, particularly TBI and lower limb injuries, compared to their younger counterparts due to a combination of increased rates of osteoporosis, decreased reaction times and reduced homeostatic reserve. ${ }^{3,31}$

Injuries in older adults after low-level falls can be severe, equivalent to the injury severity score of some younger adults who have suffered road traffic accidents. In a recent study, two-thirds of TBI-related hospital admissions were for ground-level falls, with the highest incidence of $74 \%$ in the over-85 group. ${ }^{32}$ Other studies show anticoagulant therapy is associated with poorer outcomes after TBI, including increased intracranial bleeding, longer hospital stay and greater in-hospital mortality. ${ }^{33}$

Besides increasing the risk of falls, frailty has been associated with increased risk of thrombosis and bleeding. In the Cardiovascular Health Study, frailty was found to be associated with increased risk of idiopathic venous thromboembolism. ${ }^{34,35}$ A study from Australia investigated the impact of frailty on clinical outcomes in 220 older people with AF acutely admitted to hospital. After 6-month follow-up, participants with frailty had a small nonsignificant increase in the rate of major or severe hemorrhage, but significantly higher rates of stroke and death compared with those without frailty. ${ }^{36}$ In an observational study of 235 frail older patients taking warfarin for AF, the annual rate of major hemorrhage was high at around $10 \%$ but, excluding fatalities, no long-term sequelae were observed (the annual stroke rate on warfarin was low at $2.9 \%$ ). ${ }^{35}$ Together, these studies show that although bleeding risk is increased, the risk of thrombosis in frail older patients may be even greater in some circumstances.

Where possible if the thrombotic risk is greater than the bleeding risk, oral anticoagulation (not aspirin) is still the best therapy for stroke prevention. ${ }^{37}$ There is a need for further work to explore the balance of prothrombotic and antithrombotic factors in frail and non-frail older people, and to investigate if a laboratory measure of thrombotic potential can help identify those at highest risk of thrombosis. ${ }^{38}$

\section{Major bleeding and coagulopathy in trauma}

After major trauma, uncontrolled bleeding is one of the leading causes of early mortality. ${ }^{39}$ Hemorrhage also increases the risk of multi-organ failure. ${ }^{40}$ Bleeding is a potentially preventable cause of death, and better ways to diagnose and manage acute bleeding and coagulopathy are the focus of much research. These have been translated into clinical practice where timely identification of the sources of bleeding, prompt measures to minimize blood loss, restoration of tissue perfusion and use of clotting factors appear key to hemostatic control. ${ }^{39}$

Following severe injury, patients may have impaired coagulation (trauma-induced coagulopathy) caused by the injury itself. In younger patients, this coagulopathy is seen in up to a quarter of cases and is associated with increased risk of bleeding and death. ${ }^{41}$ It is a complex process characterized by endothelial dysfunction, low fibrinogen, fibrinolysis (increased clot breakdown) and platelet dysfunction. ${ }^{42}$

Impaired hemostasis may also be due to pre-injury antithrombotic use leading to a "pharmacological coagulopathy". As a potentially reversible risk factor, identifying patients taking anticoagulants and timely reversal can minimize bleeding and associated complications, particularly in the elderly who are more likely to be taking these medications.

\section{Trauma and antithrombotics Does the type of anticoagulation or antiplatelet agent affect bleeding outcomes?}

With DOACs being more widely prescribed as an alternative to warfarin, the question arises whether there are differences in trauma outcomes in patients taking a DOAC or warfarin. An observational study on a cohort of 114 patients with severe blunt trauma (severe TBI excluded) showed DOAC use was associated with significantly lower mortality (DOAC group $8.3 \%$ vs. warfarin group $29.5 \%, p<0.015$ ). The ratio of units transfused per patient was also lower in the DOAC group (2.8 \pm 1.8 units/patient in the DOAC group vs. $6.7 \pm$ 6.4 units per patient in the warfarin group; $p=0.001) .{ }^{43}$ The same group compared outcomes in 161 patients taking either a DOAC or warfarin prior to traumatic intracranial hemorrhage. In this single-center retrospective study, the authors reported improved mortality and reduced rates of operative intervention associated with DOAC use in patients with blunt intracranial hemorrhage when compared to a similar group taking warfarin..$^{44}$

In a retrospective study of less severely injured patients, there was no difference in mortality and rate of blood transfusions between the warfarin and DOAC group..$^{45}$ Although the studies described are relatively small and non-randomized, and took place before the antidote for dabigatran was 
licensed, they do allay some of the initial fears associated with the DOACs about the lack of a specific reversal agent. A clearer picture regarding the safety of DOACs is expected as data in trauma registries mature.

A single-center observational study from Canada of 1121 older trauma patients with low injury severity (aged 65 and above) aimed to determine the effect of anticoagulation (including antiplatelets) on outcomes after falls. ${ }^{30} \mathrm{Of}$ the $30.0 \%$ of patients taking some form of anticoagulation as a home medication, patients on clopidogrel had the highest mortality compared with those on aspirin or warfarin. It is hypothesized that inferior survival in patients taking clopidogrel may be due to the lack of effective reversal agents.

The wider literature shows very little high-quality evidence to support platelet transfusion for bleeding in patients taking antiplatelet therapy. Although not in the context of traumatic bleeding, the PATCH (platelet transfusion versus standard care after acute stroke due to spontaneous cerebral hemorrhage associated with antiplatelet therapy) randomized controlled trial (RCT) was designed to answer the question whether platelet transfusions were better than standard care (ie, no platelet transfusion) in spontaneous intracranial hemorrhage. ${ }^{46}$ Two hundred patients were randomized, and the results showed the odds of death or dependence at 3 months were higher in the platelet transfusion group than in the standard care group (adjusted common odds ratio 2.05, 95\% CI 1.18-3.56; $p=0.0114)$. Twenty-three (24\%) participants assigned to platelet transfusion and $16(17 \%)$ assigned to standard care died during hospital stay. Although these findings are from a single study, they do remind clinicians about the risks of harm with the use of (biological) agents that promote bleeding control.

\section{Traumatic hemorrhage: acute management of anticoagulant and antiplatelet therapy Trauma team and general hemostatic measures}

The successful management of traumatic bleeding depends on the expertise of a multidisciplinary trauma team, although it is less clear whether separate pathways should apply for older patients. In recognition of the differences in trauma and care of the older persons, there are some proponents of trauma center providing dedicated set-up to care for older people. Such trauma pathways for the older persons with trauma ${ }^{47}$ could particularly draw attention to management of delirium and reversal of anticoagulation effects.

Management of a bleeding patient should include general supportive measures such as applying pressure, tourniquet and elevation. There is no reason to omit the antifibrinolytic drug tranexamic acid within 3 hours of injury, ${ }^{48,49}$ although it could be questioned whether sufficient safety data exist for those patients at extremes of old age. A history of anticoagulation or antiplatelet therapy may not be readily available (eg, due to delirium or head injury), but past records and collateral history may be helpful, so prompt reversal therapy can be administered to prevent worsening of bleeding. Damage control resuscitation describes the principles of acute hemorrhage care with hemostatic resuscitation (blood product to aid perfusion and hemostasis) and definitive control of bleeding through radiological or surgical intervention. Maintaining perfusion and supporting blood pressure will also aid renal perfusion and function, particularly important for DOAC excretion.

For minor bleeding or where there is no bleeding and no emergent need for anticoagulant reversal, options include discontinuing anticoagulation and delaying any surgery/ procedures until the anticoagulant effect has subsided. In the frail, elderly patients, with comorbidities, it is also important to consider if renal impairment is present, as it will prolong the half-lives of the DOACs.

\section{Managing warfarin-related major bleeding}

Early assessment and identification of anticoagulation is an important determinant of outcomes in trauma patients. Guidelines on the reversal of anticoagulation have been written for the general adult population, although there are no specific guidelines for older people. For emergency reversal of vitamin $\mathrm{K}$ antagonists, intravenous vitamin $\mathrm{K}$ and 4-factor prothrombin complex concentrate (PCC) are recommended. ${ }^{12-14}$ PCC is superior to fresh frozen plasma (FFP), and FFP is not recommended for warfarin reversal if PCC is available. ${ }^{12,14}$ Timely and effective reversal is paramount, and proper doses of vitamin $\mathrm{K}$ and PCC should be given to reverse warfarin (Table 1). After the acute event, bridging of anticoagulation can be covered with low-molecular weight heparin if needed.

\section{Managing DOAC-related bleeding}

In the acute setting, in the presence of major or life-threatening bleeding, DOAC reversal should be considered. Dabigatran is primarily renally excreted, and there is now an effective antidote idarucizumab, a humanized antibody fragment that 
Table I Commonly prescribed anticoagulation and antiplatelet therapy, their mechanisms of action and strategies for emergency control of bleeding/reversal

\begin{tabular}{|c|c|c|c|}
\hline Drug & Mechanism of action & Half-life & Emergency reversal \\
\hline \multirow[t]{2}{*}{ Warfarin } & Vitamin $\mathrm{K}$ antagonist & $20-60$ hours & Vitamin K 5 g IV \\
\hline & & & PCC, dose approximately $25-50$ units $/ \mathrm{kg}$ \\
\hline $\begin{array}{l}\text { Direct oral } \\
\text { anticoagulants }\end{array}$ & Mechanism of action & Half-life & Emergency reversal \\
\hline Dabigatran & Thrombin inhibitor & $1 \mathrm{I}-13$ hours (dependent on renal function) & Idarucizumab antidote - 5 g IV \\
\hline Rivaroxaban & Factor $\mathrm{Xa}$ inhibitor & $\sim 9$ hours (dependent on renal function) & $\begin{array}{l}\text { No specific antidote licensed yet, but } \\
\text { andexanet alfa is in late clinical trials }\end{array}$ \\
\hline Apixaban & Factor $\mathrm{Xa}$ inhibitor & & Current options include PCC and \\
\hline Edoxaban & Factor $\mathrm{Xa}$ inhibitor & & activated PCC \\
\hline Antiplatelet & Mechanism of action & $\begin{array}{l}\text { Time to normal platelet function after } \\
\text { drug cessation }\end{array}$ & $\begin{array}{l}\text { Therapeutic options for emergency } \\
\text { control of bleeding }\end{array}$ \\
\hline Aspirin & Irreversibly inhibits COXI & $5-7$ days & Platelet transfusion (low-level evidence) \\
\hline Clopidogrel & Irreversibly inhibits $\mathrm{P} 2 \mathrm{Y} 12$ receptors & $5-7$ days & Desmopressin (caution with \\
\hline Prasugrel & Irreversibly inhibits $\mathrm{P} 2 \mathrm{Y} 12$ receptors & 5-7 days & contraindications and limited evidence \\
\hline Ticagrelor & Reversibly inhibits $\mathrm{P} 2 \mathrm{Y} 12$ receptors & $3-5$ days & in trauma) \\
\hline Dipyridamole & Phosphodiesterase inhibitor & 24 hours & \\
\hline
\end{tabular}

Notes: Data from Kehoe et al," Keeling et al, ${ }^{12}$ and Hunt et al. ${ }^{13}$

Abbreviations: IV, intravenous; PCC, prothrombin complex concentrate.

binds specifically to dabigatran. This means there should be less need for hemodialysis as a management strategy.

In situations of ongoing life-threatening bleeding in the presence of a factor $\mathrm{Xa}$, where there are no specific antidotes, a non-specific procoagulant such as PCC or activated PCC has been used. Activated PCC may carry a greater risk of thrombosis, but may be considered if inactive 4-factor PCC is not available. Recombinant factor VIIa should not be a firstchoice option due to lack of efficacy data and the potential for thrombotic complications, which may be greater in the older trauma patients. ${ }^{50}$

The risk/benefit profile of a procoagulant agent should be assessed for each case. Antidote development for the factor Xa inhibitors is in progress. Andexanet is a recombinant human factor Xa decoy protein which neutralizes the effect of factor Xa inhibitors and has demonstrated hemostatic efficacy in the clinical trial setting, but is not yet approved for general use. ${ }^{51,52} \mathrm{~A}$ second antidote ciraparantag works by binding to a range of anticoagulants by charge-charge interaction and removing them from their target, and is in late-phase development. ${ }^{53}$

The British Society of Hematology on Perioperative Management of Anticoagulation and Antiplatelet Therapy recommends, when possible, for patient taking DOACs without emergent reversal, surgery should be delayed to allow the plasma level of the drug to fall. ${ }^{54}$ The concentration of drug can be estimated from the dose of the drug, time of the last dose and the patient's renal function. Other factors, such as patient sex, weight and the use of interacting drugs, will also have less significant effects. ${ }^{54}$

\section{Managing antiplatelet-related major bleeding}

Antiplatelet drugs have short plasma half-lives but may have a prolonged biological effect due to irreversible platelet inhibition. The average lifespan of a platelet is approximately 7 days, and only when new platelets are made will the effect of the drug wear off. ${ }^{55}$ As there are no specific reversal agents for antiplatelets, the treatment of bleeding requires general measures for controlling hemostasis, cessation of antiplatelet treatment and reversal of co-prescribed anticoagulant. ${ }^{55}$

For emergency reversal of aspirin, an infusion of 2-3 adult doses of donor platelets is usually effective. In vitro studies and a case report suggest that platelet dysfunction caused by aspirin is much easier to correct with platelet transfusion compared with clopidogrel. ${ }^{55}$ The efficacy of platelet transfusion may be reduced in patients taking clopidogrel, and a higher dose of platelets may be needed.

There has been interest in other agents to support coagulation. Desmopressin, a synthetic vasopressor, reduced the bleeding time in healthy volunteers after exposure to clopidogrel, but safety concerns in patients with cardiovascular disease may restrict use, particularly in the older population where cardiovascular disease is more prevalent. ${ }^{55}$ In the trauma setting, the European Trauma Guidelines suggest the 
use of desmopressin $(0.3 \mu / \mathrm{kg})$ for patients with bleeding with Von Willebrand disease or those taking antiplatelet therapy. ${ }^{39}$

In addition to stopping the source of bleeding and general hemostatic measures, tranexamic acid is also suggested to counteract the effect of antiplatelet agents after risk/benefit assessment. ${ }^{55}$ An approach to the acute management of bleeding in the older persons with trauma is given in Figure 1.

Assessment of injury/bleeding risk/emergent surgery
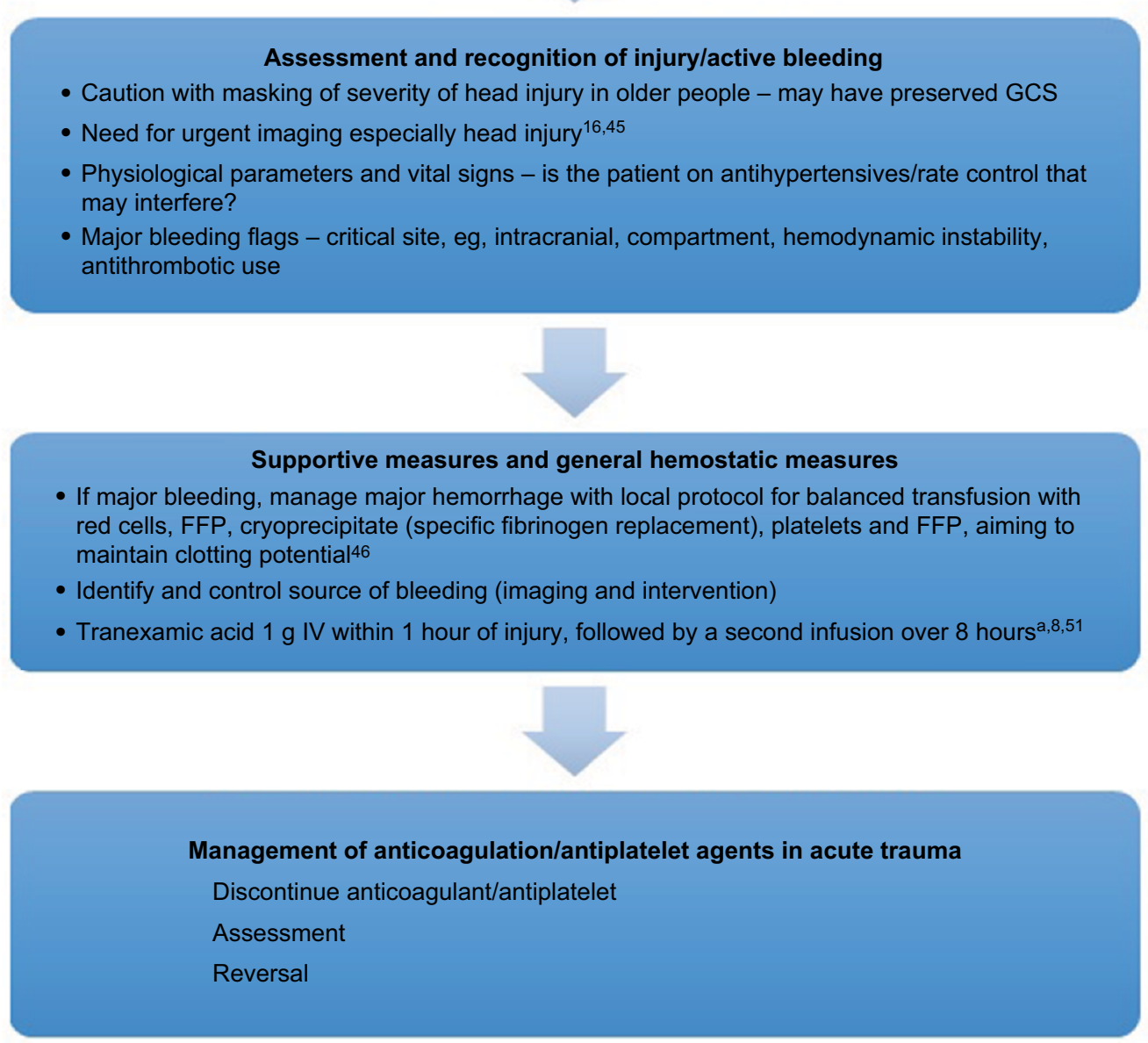

\section{Assessment of effect of antithrombotic}

- Type of antithrombotic, last dose - timing and dose taken

- Assessment of coagulation, hematology, renal function. Point of care INR if available

- Laboratory tests, eg, INR, APTT, PT, fibrinogen (specific assays of DOAC may not be available)

\section{Reversal}

- Consider indications for reversal, eg, major bleeding

- If reversal indicated use specific agents where possible

- For immediate reversal of warfar in - vitamin $\mathrm{K}$ 5-10 mg IV and PCC

- For immediate reversal of dabigatran - idarucizumab $5 \mathrm{mg}$ IV

- Rivaroxaban, apixaban, edoxaban antidote in development (andexanet alfa). Options currently include PCC and activated PCC. Caution with activated PCC use as increased risk of thrombosis

- For antiplatelets, no specific antidote. Options include platelet transfusion, desmopressin ${ }^{14,39}$

Figure I Acute management of the older trauma patients with bleeding.

Notes: aCRASH-2 trial showed TXA is most effective within I hour of injury and harmful after 3 hours.

Abbreviations: GCS, Glasgow Coma Scale; FFP, fresh frozen plasma; INR, international normalized ratio; APTT, activated partial thromboplastin time; PT, prothrombin time; DOAC, direct oral anticoagulant; IV, intravenous; PCC, prothrombin complex concentrate; TXA, tranexamic acid. 


\section{Antithrombotics: decision making after trauma}

Once hemostasis has been achieved, longer-term decisions regarding recommencing antithrombotics can be complex. Weighing up the balance between prothrombotic risk and falls or bleeding risk can often be challenging and compounded by a paucity of evidence to guide decisions regarding reinstitution of antithrombotics. Many of the initial DOAC trials did not include frail older patients with multiple comorbidities. However, the sharing of data from ongoing registries is vital in understanding real-world data on bleeding incidence and outcomes in patients on DOACs. Recommencing anticoagulation risks early rebleeding and recurrence in the face of future trauma. Conversely, failure to re-anticoagulate can leave the patient at risk of thrombosis. ${ }^{56}$ It is important to consider the cause of the trauma and if it is likely to reoccur along with the initial reason for anticoagulation and if this is still strongly indicated. High-risk indications for anticoagulation such as mechanical heart valve and antiphospholipid disease with recurrent thromboembolic events require the resumption of some sort of anticoagulation. It is important to take an individualized approach, and a form of bridging therapy such as intravenous heparin or low-molecular weight heparin may be considered as an interim measure. In AF, well-established risk prediction tools including the $\mathrm{CHA}_{2} \mathrm{DS}_{2}$ VASc and the HASBLED scores can aid weighing up the risk/benefit ratio of anticoagulation. It is also important to consider that trauma itself, surgery and immobility also heighten the hypercoagulable state.
Although serious consequences, for example, subdural hematoma (SDH) and intracranial hemorrhage, are rare, they can have devastating effects and are therefore likely to influence decision making regarding anticoagulation for stroke prevention in patients with AF. It has been estimated that approximately $33 \%$ of patients over 65 years fall per year and the rate of SDH among this group is 0.0004 for every patient year. ${ }^{57} \mathrm{~A}$ study by Man-Son-Hing et al used a Markov decision analytical model to determine the optimal anticoagulation strategy for patients with AF over 65 years who had a risk of falling. ${ }^{57}$ Here, they compared patients on warfarin, aspirin and no therapy. They demonstrated that among patients with an average risk of AF and falls, warfarin therapy was associated with quality life-adjusted years of 12.90 , aspirin therapy 11.17 and no therapy 10.15 . They also calculated that the risk of an SDH must be 535 times greater for the risks of warfarin therapy to outweigh the benefits. In their model, given that the average person over 65 years has around 1.8 falls a year, they have estimated that a person taking warfarin needs to fall 295 times a year before warfarin is not regarded as the optimal therapy.

Decisions regarding resumption of anticoagulation are complex and should be taken on a case-by-case basis and involve a holistic approach (Figure 2). A multidisciplinary approach is often helpful, including involvement from the patient, family/carers, therapists, general practitioner and specialists. If a fall were the cause of the injury, review by a falls specialist is recommended; evidence-based interventions do reduce the risk of future falls.
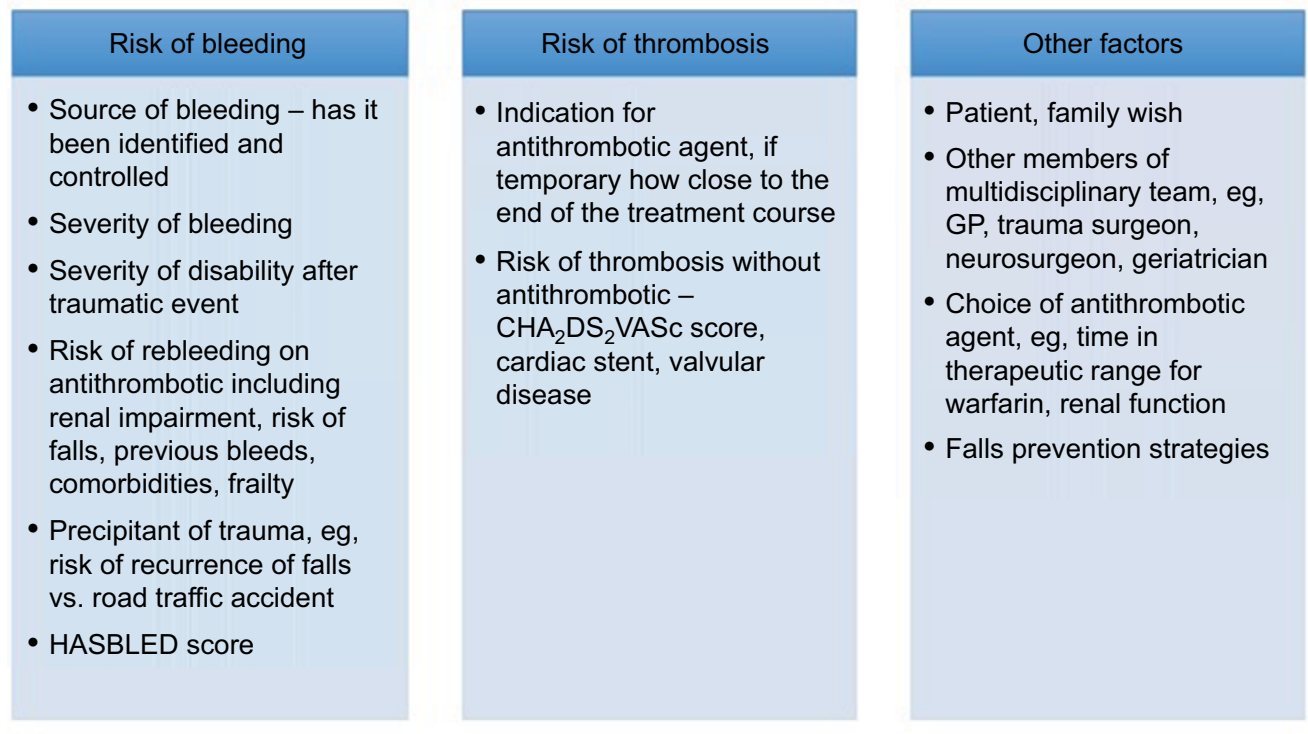

Figure 2 Decisions regarding anticoagulation/antiplatelet resumption: factors to consider. Abbreviation: GP, general practitioner. 


\section{Resuming anticoagulation after TBI}

The optimal timing for resumption of anticoagulation after TBI is uncertain. Retrospective studies have investigated outcomes after anticoagulation resumption following intracranial hemorrhage. Albrecht et al estimated the risk of thrombotic and hemorrhagic events as a function of the timing of anticoagulant therapy resumption following TBI in a retrospective study of 10,782 patients. ${ }^{58}$ Following hospital discharge, $55 \%$ had taken warfarin during at least 1 or more 30-day periods prior to TBI. Warfarin use in the prior period was associated with decreased risk of thrombotic events (relative risk [RR], 0.77 [95\% CI, 0.67-0.88]) and increased risk of hemorrhagic events (RR, 1.51 [95\% CI, 1.29-1.78]). However, there was an overall net benefit in recommencing warfarin; warfarin use was associated with decreased risk of hemorrhagic or ischemic stroke (RR, 0.83 [95\% CI, 0.72-0.96]). ${ }^{58}$

In the setting of spontaneous intracranial hemorrhage, data from a retrospective study also support resumption of anticoagulation. ${ }^{59}$ Oral anticoagulation (including antiplatelet therapy) was restarted in 172 of 719 (23.9\%) patients following spontaneous bleeding. Median time to anticoagulation resumption was 31 days. Anticoagulation therapy resumption was associated with fewer ischemic events (anticoagulation 9/172 [5.2\%]; no anticoagulation 82/547 [15.0\%]; $p<0.001$ ), without significant increase in hemorrhagic complications.

More recently, a retrospective study of 72 patients showed that no TBI cases developed serious neurologic deterioration related to the administration of anticoagulation within 60 days after injury ${ }^{60}$ However, in the subset of patients with repeat $\mathrm{CT}$, older age (age 65 and above) was a risk factor for worsening hemorrhage on repeat scan. ${ }^{60}$

Despite these data, deciding whether or not to recommence antithrombotics can be difficult, and there is no randomized evidence to guide decision making. For spontaneous intracranial hemorrhage, based on data from observational studies, the European Stroke Initiative suggest first rechecking the indication for anticoagulation. ${ }^{61}$ They suggest oral antithrombotic therapy may be continued after 10-14 days, depending on the perceived risk of thromboembolic occlusion and intracranial bleed recurrence. ${ }^{61}$ In practice, deciding when to restart anticoagulation can be difficult, and hence, expertise from the multidisciplinary team should be sought. A detailed review of TBI coagulopathy and hemorrhage diagnosis and management dilemmas has recently been published by Maegele et al. ${ }^{62}$

We await results of the RESTART RCT, which is testing whether a policy of starting antiplatelet drugs after spontaneous intracranial bleeding results in a beneficial net reduction of serious vascular event years compared with a policy of avoiding antiplatelet drugs (currently recruiting patients, ISRCTN71907627).

\section{Falls prevention strategies}

Decisions regarding anticoagulants should involve a holistic approach that includes strategies to prevent injury. The majority of falls in the elderly are caused by interplay of many different factors, and assessment of these patients should be holistic with the aim of identifying and modifying the contributory factors. A systematic review and meta-analysis demonstrated that a multifactorial falls risk assessment and intervention program significantly reduced the risk of falling (adjusted risk ratio, 0.82 [95\% CI, 0.72-0.94], number needed to treat 11) and monthly falls rate (adjusted risk ratio, 0.63 [95\% CI, 0.49-0.83]). ${ }^{63}$ The most frequent factors assessed were vision, home environmental hazards, orthostatic blood pressure and medication.

\section{Case review}

In the case study set out at the beginning of this article, the patient's mechanism of injury and symptoms are concerning for potential TBI and risk of hemorrhage as she is taking warfarin. (It is important to also consider other causes of confusion, for example, infection.) Emergency reversal of warfarin with PCC and vitamin $\mathrm{K}$ is indicated in critical site or major bleeding. We would measure the prothrombin time/ international normalized ratio as soon as possible and arrange an urgent head CT scan. As there is a strong suspicion of intracerebral bleed, we would reverse the warfarin before the results of any investigations, in keeping with current guidelines. ${ }^{12}$ In the longer term, our patient would be reviewed by the falls team, and our discussions with the patient regarding anticoagulation and decision making would be based on the factors described in Figure 2.

\section{Conclusion}

There is increasing recognition of the complexities of management of an older, frail person with trauma. Comorbidities and physiological changes that occur with ageing can contribute to the challenges associated with recognition and management of bleeding in older patients. Although substantial improvements have been made across trauma care over the past decades, a change in trauma demographics shows the particular needs of older people have yet to be met. Further study is needed to guide decision making regarding initiation or reintroduction of antithrombotics, particularly how we can 
identify the older person whose risk of falling with subsequent major bleeding, traumatic complications and death is greater than the annual risk of death or disability due to stroke off antithrombotics. ${ }^{64}$ There is also a need to better understand the mechanisms of pharmacological and trauma-induced coagulopathy in the elderly, so we can improve treatment and outcomes particularly in the management of antiplateletrelated bleeding. Specific patient pathways for older patients with trauma, incorporating integrated trauma and geriatric care, should be considered in larger trauma centers.

\section{Acknowledgment}

The authors would like to thank Claire Pulford and Mary Clements for their helpful advice on this topic.

\section{Disclosure}

The authors report no conflicts of interest in this work.

\section{References}

1. Soong J, Poots AJ, Scott S, et al. Quantifying the prevalence of frailty in English hospitals. BMJ Open. 2015;5(10):e008456.

2. National Institute for Health and Care Excellence. Major trauma: assessment and initial management. NICE Guideline [NG39]. 2016.

3. TARN. TARN 2017 report: major trauma in older people. 2017. Available from: https://www.ons.gov.uk/peoplepopulationandcommunity/ populationandmigration/populationestimates/articles/overviewoftheukpopulation/mar2017. Accessed September 7, 2017.

4. Cooper Z, Maxwell CA, Fakhry SM, et al. A position paper: the convergence of aging and injury and the need for a Geriatric Trauma Coalition (GeriTraC). J Trauma Acute Care Surg. 2017;82(2):419-422.

5. Kozar RA, Arbabi S, Stein DM, et al. Injury in the aged: geriatric trauma care at the crossroads. J Trauma Acute Care Surg. 2015;78(6): 1197-1209.

6. Ohmori T, Kitamura T, Tanaka K, et al. Bleeding sites in elderly trauma patients who required massive transfusion: a comparison with younger patients. Am J Emerg Med. 2016;34(2):123-127.

7. Office for National Statistics. Overview of the UK population: March 2017. Available from: https://www.ons.gov.uk/peoplepopulationandcommunity/populationandmigration/populationestimates/articles/ overviewoftheukpopulation/mar2017. Accessed September 7, 2017.

8. Hashmi A, Ibrahim-Zada I, Rhee P, et al. Predictors of mortality in geriatric trauma patients: a systematic review and meta-analysis. $J$ Trauma Acute Care Surg. 2014;76(3):894-901.

9. Salottolo KM, Mains CW, Offner PJ, Bourg PW, Bar-Or D. A retrospective analysis of geriatric trauma patients: venous lactate is a better predictor of mortality than traditional vital signs. Scand JTrauma Resusc Emerg Med. 2013;21:7.

10. Kehoe A, Rennie S, Smith JE. Glasgow Coma Scale is unreliable for the prediction of severe head injury in elderly trauma patients. Emerg Med J. 2015;32(8):613-615.

11. Kehoe A, Smith JE, Bouamra O, Edwards A, Yates D, Lecky F. Older patients with traumatic brain injury present with a higher GCS score than younger patients for a given severity of injury. Emerg Med J. 2016;33(6):381-385

12. Keeling D, Baglin T, Tait C, et al; British Committee for Standards in Haematology. Guidelines on oral anticoagulation with warfarin - fourth edition. Br J Haematol. 2011;154(3):311-324.

13. Hunt BJ, Allard S, Keeling D, Norfolk D, Stanworth SJ, Pendry K; British Committee for Standards in Haematology. A practical guideline for the haematological management of major haemorrhage. $\mathrm{Br}$ J Haematol. 2015;170(6):788-803.
14. Makris M, Van Veen JJ, Tait CR, Mumford AD, Laffan M; British Committee for Standards in Haematology. Guideline on the management of bleeding in patients on antithrombotic agents. Br J Haematol. 2013;160(1): $35-46$.

15. Go AS, Hylek EM, Phillips KA, et al. Prevalence of diagnosed atrial fibrillation in adults: national implications for rhythm management and stroke prevention: the AnTicoagulation and Risk Factors in Atrial Fibrillation (ATRIA) Study. JAMA. 2001;285(18):2370-2375.

16. Scowcroft AC, Lee S, Mant J. Thromboprophylaxis of elderly patients with AF in the UK: an analysis using the General Practice Research Database (GPRD) 2000-2009. Heart. 2013;99(2):127-132.

17. Hart RG, Pearce LA, Aguilar MI. Meta-analysis: antithrombotic therapy to prevent stroke in patients who have nonvalvular atrial fibrillation. Ann Intern Med. 2007;146(12):857-867.

18. Ruff CT, Giugliano RP, Braunwald E, et al. Comparison of the efficacy and safety of new oral anticoagulants with warfarin in patients with atrial fibrillation: a meta-analysis of randomised trials. Lancet. 2014;383(9921):955-962.

19. Gómez-Outes A, Terleira-Fernández AI, Calvo-Rojas G, Suárez-Gea ML, Vargas-Castrillón E. Direct oral anticoagulants for stroke prevention in patients with atrial fibrillation: meta-analysis by geographic region with a focus on European patients. Br J Clin Pharmacol. 2016;82(3): 633-644.

20. National Institute for Health and Care Excellence. Atrial fibrillation: management. Clinical Guideline [CG180]. 2014.

21. Li L, Geraghty OC, Mehta Z, Rothwell PM; Oxford Vascular Study. Age-specific risks, severity, time course, and outcome of bleeding on long-term antiplatelet treatment after vascular events: a populationbased cohort study. Lancet. 2017;390(10093):490-499.

22. Williams CD, Chan AT, Elman MR, et al. Aspirin use among adults in the U.S.: results of a national survey. Am J Prev Med. 2015;48(5): 501-508.

23. Katz DF, Maddox TM, Turakhia M, et al. Contemporary trends in oral anticoagulant prescription in atrial fibrillation patients at low to moderate risk of stroke after guideline-recommended change in use of the CHADS2 to the CHA2DS2-VASc score for thromboembolic risk assessment: analysis from the national cardiovascular data registry's outpatient practice innovation and clinical excellence atrial fibrillation registry. Circ Cardiovasc Qual Outcomes. 2017;10(5). pii:e003476.

24. Calland JF, Ingraham AM, Martin N, et al; Eastern Association for the Surgery of Trauma. Evaluation and management of geriatric trauma: an Eastern Association for the Surgery of Trauma practice management guideline. J Trauma Acute Care Surg. 2012;73(5 Suppl 4):S345-S350.

25. Joseph B, Pandit V, Zangbar B, et al. Superiority of frailty over age in predicting outcomes among geriatric trauma patients: a prospective analysis. JAMA Surg. 2014;149(8):766-772.

26. Fried LP, Tangen CM, Walston J, et al; Cardiovascular Health Study Collaborative Research Group. Frailty in older adults: evidence for a phenotype. J Gerontol A Biol Sci Med Sci. 2001;56(3):M146-M157.

27. Rockwood K, Song X, MacKnight C, et al. A global clinical measure of fitness and frailty in elderly people. CMAJ. 2005;173(5):489-495.

28. British Geriatrics Society. Fit for Frailty Part 1. Consensus best practice guidance for the care of older people living in community and outpatient settings. 2014. Available from: http://www.bgs.org.uk/campaigns/fff/ fff_full.pdf. Accessed August 16, 2017.

29. Clegg A, Young J, Iliffe S, Rikkert MO, Rockwood K. Frailty in elderly people. Lancet. 2013;381(9868):752-762.

30. Coleman J, Baldawi M, Heidt D. The effect anticoagulation status on geriatric fall trauma patients. Am J Surg. 2016;212(6):1237-1242.

31. Lee H, Bein KJ, Ivers R, Dinh MM. Changing patterns of injury associated with low-energy falls in the elderly: a 10-year analysis at an Australian Major Trauma Centre. ANZ J Surg. 2015;85(4):230-234.

32. Haring RS, Narang K, Canner JK, et al. Traumatic brain injury in the elderly: morbidity and mortality trends and risk factors. J Surg Res. 2015;195(1):1-9.

33. Dams-O'Connor K, Cuthbert JP, Whyte J, Corrigan JD, Faul M, Harrison-Felix C. Traumatic brain injury among older adults at level I and II trauma centers. J Neurotrauma. 2013;30(24):2001-2013. 
34. Folsom AR, Boland LL, Cushman M, Heckbert SR, Rosamond WD, Walston JD. Frailty and risk of venous thromboembolism in older adults. J Gerontol A Biol Sci Med Sci. 2007;62(1):79-82.

35. Johnson CE, Lim WK, Workman BS. People aged over 75 in atrial fibrillation on warfarin: the rate of major hemorrhage and stroke in more than 500 patient-years of follow-up. JAm Geriatr Soc. 2005;53(4): 655-659.

36. Perera V, Bajorek BV, Matthews S, Hilmer SN. The impact of frailty on the utilisation of antithrombotic therapy in older patients with atrial fibrillation. Age Ageing. 2009;38(2):156-162.

37. Tay KH, Lane DA, Lip GY. Challenges facing anticoagulation among the elderly and frail. Age Ageing. 2009;38(2):140-142.

38. Nguyen TN, Morel-Koop MC, Pepperell D, Cumming RG, Hilmer $\mathrm{SN}$, Ward CM. The impact of frailty on coagulation and responses to warfarin in acute older hospitalised patients with atrial fibrillation: a pilot study. Aging Clin Exp Res. Epub 2017 Mar 2.

39. Rossaint R, Bouillon B, Cerny V, et al. The European guideline on management of major bleeding and coagulopathy following trauma: fourth edition. Crit Care. 2016;20:100.

40. Cohen MJ, Call M, Nelson M, et al. Critical role of activated protein $\mathrm{C}$ in early coagulopathy and later organ failure, infection and death in trauma patients. Ann Surg. 2012;255(2):379-385.

41. Brohi K, Singh J, Heron M, Coats T. Acute traumatic coagulopathy. $J$ Trauma Acute Care Surg. 2003;54(6):1127-1130.

42. Davenport RA, Brohi K. Cause of trauma-induced coagulopathy. Curr Opin Anaesthesiol. 2016;29(2):212-219.

43. Feeney JM, Neulander M, DiFiori M, et al. Direct oral anticoagulants compared with warfarin in patients with severe blunt trauma. Injury. 2017;48(1):47-50.

44. Feeney JM, Santone E, DiFiori M, Kis L, Jayaraman V, Montgomery SC. Compared to warfarin, direct oral anticoagulants are associated with lower mortality in patients with blunt traumatic intracranial hemorrhage: a TQIP study. J Trauma Acute Care Surg. 2016;81(5):843-848.

45. Barletta JF, Hall S, Sucher JF, Dzandu JK, Haley M, Mangram AJ. The impact of pre-injury direct oral anticoagulants compared to warfarin in geriatric G-60 trauma patients. Eur J Trauma Emerg Surg. 2017;43(4):445-449.

46. Baharoglu MI, Cordonnier C, Al-Shahi Salman R, et al; PATCH Investigators. Platelet transfusion versus standard care after acute stroke due to spontaneous cerebral haemorrhage associated with antiplatelet therapy (PATCH): a randomised, open-label, phase 3 trial. Lancet. 2016;387(10038):2605-2613.

47. Zafar SN, Obirieze A, Schneider EB, et al. Outcomes of trauma care at centers treating a higher proportion of older patients: the case for geriatric trauma centers. J Trauma Acute Care Surg. 2015;78(4): 852-859.

48. CRASH-2 collaborators, Roberts I, Shakur H, Afolabi A, et al. The importance of early treatment with tranexamic acid in bleeding trauma patients: an exploratory analysis of the CRASH-2 randomised controlled trial. Lancet. 2011;377(9771):1096-1101, 1101.e1-e2.
49. CRASH-2 trial collaborators, Shakur H, Roberts I, Bautista R, et al. Effects of tranexamic acid on death, vascular occlusive events, and blood transfusion in trauma patients with significant haemorrhage (CRASH2): a randomised, placebo-controlled trial. Lancet. 2010;376(9734): 23-32.

50. Steiner T, Weitz JI, Veltkamp R. Anticoagulant-associated intracranial hemorrhage in the era of reversal agents. Stroke. 2017;48(5):1432-1437.

51. Siegal DM, Curnutte JT, Connolly SJ, et al. Andexanet alfa for the reversal of factor Xa inhibitor activity. N Engl J Med. 2015;373(25):2413-2424.

52. Connolly SJ, Milling TJ Jr, Eikelboom JW, et al; ANNEXA-4 Investigators. Andexanet alfa for acute major bleeding associated with factor Xa inhibitors. N Engl J Med. 2016;375(12):1131-1141.

53. Ansell JE, Bakhru SH, Laulicht BE, et al. Single-dose ciraparantag safely and completely reverses anticoagulant effects of edoxaban. Thromb Haemost. 2017;117(2):238-245.

54. Keeling D, Tait RC, Watson H; British Committee of Standards for Haematology. Peri-operative management of anticoagulation and antiplatelet therapy. Br J Haematol. 2016;175(4):602-613.

55. Estcourt LJ, Birchall J, Allard S, et al; British Committee for Standards in Haematology. Guidelines for the use of platelet transfusions. $\mathrm{Br} J$ Haematol. 2017;176(3):365-394.

56. Saccullo G, Bucko AM, Van Veen JJ, Makris M. Why is it so difficult to show that prothrombin complex concentrates are superior to fresh-frozen plasma for warfarin reversal? Blood Transfus. 2017;15(3):277-278.

57. Man-Son-Hing M, Nichol G, Lau A, Laupacis A. Choosing antithrombotic therapy for elderly patients with atrial fibrillation who are at risk for falls. Arch Intern Med. 1999;159(7):677-685.

58. Albrecht JS, Liu X, Baumgarten M, et al. Benefits and risks of anticoagulation resumption following traumatic brain injury. JAMA Intern Med. 2014;174(8):1244-1251.

59. Kuramatsu JB, Gerner ST, Schellinger PD, et al. Anticoagulant reversal, blood pressure levels, and anticoagulant resumption in patients with anticoagulation-related intracerebral hemorrhage. JAMA. 2015;313(8):824-836.

60. Matsushima $\mathrm{K}$, Inaba $\mathrm{K}$, Cho J, et al. Therapeutic anticoagulation in patients with traumatic brain injury. J Surg Res. 2016;205(1):186-191.

61. Steiner T, Kaste M, Forsting M, et al. Recommendations for the management of intracranial haemorrhage - part I: spontaneous intracerebral haemorrhage. The European Stroke Initiative Writing Committee and the Writing Committee for the EUSI Executive Committee. Cerebrovasc Dis. 2006;22(4):294-316.

62. Maegele M, Schöchl H, Menovsky T, et al. Coagulopathy and haemorrhagic progression in traumatic brain injury: advances in mechanisms, diagnosis, and management. Lancet Neurol. 2017;16(8):630-647.

63. Chang JT, Morton SC, Rubenstein LZ, et al. Interventions for the prevention of falls in older adults: systematic review and meta-analysis of randomised clinical trials. BMJ. 2004;328(7441):680.

64. Boltz MM, Podany AB, Hollenbeak CS, Armen SB. Injuries and outcomes associated with traumatic falls in the elderly population on oral anticoagulant therapy. Injury. 2015;46(9):1765-1771.
Journal of Blood Medicine

\section{Publish your work in this journal}

The Journal of Blood Medicine is an international, peer-reviewed, open access, online journal publishing laboratory, experimental and clinical aspects of all aspect pertaining to blood based medicine including but not limited to: Transfusion Medicine; Blood collection, Donor issues, Transmittable diseases, and Blood banking logistics; Immunohematology; Artificial and alternative blood based therapeutics; Hematology; Biotechnology/nanotechnology of blood related medicine; Legal aspects of blood medicine; Historical perspectives. The manuscript management system is completely online and includes a very quick and fair peer-review system. Visit http://www.dovepress.com/ testimonials.php to read real quotes from published authors. 